\title{
The Initial and Neumann Boundary Value Problem for a Class Parabolic Monge-Ampère Equation
}

\author{
Juan Wang, ${ }^{1}$ Jinlin Yang, ${ }^{1}$ and Xinzhi Liu ${ }^{2}$ \\ ${ }^{1}$ School of Mathematics, Physics and Biological Engineering, Inner Mongolia University of Science and Technology, \\ Baotou 014010, China \\ ${ }^{2}$ Department of Applied Mathematics, University of Waterloo, Waterloo, ON, Canada N2L 3G1
}

Correspondence should be addressed to Juan Wang; tlwangjuan@126.com

Received 26 March 2013; Accepted 11 June 2013

Academic Editor: Sergey Piskarev

Copyright (C) 2013 Juan Wang et al. This is an open access article distributed under the Creative Commons Attribution License, which permits unrestricted use, distribution, and reproduction in any medium, provided the original work is properly cited.

We consider the existence, uniqueness, and asymptotic behavior of a classical solution to the initial and Neumann boundary value problem for a class nonlinear parabolic equation of Monge-Ampère type. We show that such solution exists for all times and is unique. It converges eventually to a solution that satisfies a Neumann type problem for nonlinear elliptic equation of MongeAmpère type.

\section{Introduction}

Historically, the study of Monge-Ampère is motivated by the following two problems: Minkowski and Weyl problems. One is of prescribing curvature type, and the other is of embedding type. The development of Monge-Ampère theory in PDE is closely related to that of fully nonlinear equations. Generally speaking, there are two ways to tackle the problems. One is via continuity method involving some appropriate a priori estimates, and the other is weak solution theory. MongeAmpère equations have many applications. In recent years new applications have been found in affine geometry and optimal transportation problem.

Many scholars have studied this kind of equations (see, e.g., [1-5] and the references given therein). Their main work is directed at the first or the third boundary value problem. But concerning Neumann boundary value problem, there is lack of research. In this paper, we consider the existence, uniqueness, and asymptotic behavior of a classical solution to the initial and Neumann boundary value problem for a class parabolic equation of Monge-Ampère type as follows:

$$
\begin{gathered}
\dot{u}=\operatorname{det}^{1 / n}\left(D_{x}^{2} u\right)-g_{\sigma}(x, u) \quad \text { in } \Omega \times(0, T], \\
u_{v}=\varphi(x, u) \quad \text { on } \partial \Omega \times[0, T], \\
\left.u\right|_{t=0}=u_{0} \quad \text { in } \Omega,
\end{gathered}
$$

where $\dot{u}=\partial u / \partial t$ and $\Omega$ is a bounded, uniformly convex domain in $R^{n}$ with the boundary $\partial \Omega \in C^{4+\alpha}$. $v$ denotes the unit inner normal on $\partial \Omega$ which has been extended on $\overline{Q_{T}}$ to become a properly smooth vector field independent of $t$. For some $T_{0}, T_{0} \in(0, T)$, when $t \in\left(0, T_{0}\right], g_{\sigma}(x, u)=g_{1}(x, u)$, and when $t \in\left(T_{0}, T\right], g_{\sigma}(x, u)=g_{2}(x, u)$. The function $g_{\sigma} \epsilon$ $C^{2+\alpha, 2+\alpha}(\bar{\Omega} \times R), \sigma=1,2$. For each $x \in \Omega, \lim _{t \rightarrow T_{0}^{+}} g_{2}(x, u(x, t))=$ $g_{1}\left(x, u\left(x, T_{0}\right)\right)$. Here $\varphi \in C^{3+\alpha, 3+\alpha}(\bar{\Omega} \times R)$ and $u_{0} \in C^{4+\alpha}(\bar{\Omega})$. The initial value $u_{0}$ is a strictly convex function on $\bar{\Omega}$. In the sequel we assume for simplicity that $0 \in \Omega$.

To guarantee the existence of the classical solutions for (1) and convergence to a solution with prescribed curvature, we have to assume several structure conditions analogous to [6]. These are

$$
\begin{gathered}
\varphi_{z} \equiv \frac{\partial \varphi(x, z)}{\partial z} \geq c_{\varphi}>0 \\
g_{\sigma}>0, \quad\left(g_{\sigma}\right)_{z} \equiv \frac{\partial g_{\sigma}(x, z)}{\partial z} \geq 0, \quad \text { for } \sigma=1,2 \\
\operatorname{det}^{1 / n}\left(D_{x}^{2} u_{0}\right)-g\left(x, u_{0}\right) \geq 0
\end{gathered}
$$

Moreover, we will always assume the following compatibility conditions to be fulfilled on $\partial \Omega \times\{t=0\}$ : 


$$
\begin{gathered}
\left(u_{0}\right)_{v}=\varphi\left(x, u_{0}\right), \\
\left(\operatorname{det}^{1 / n}\left(D_{x}^{2} u_{0}\right)-g_{1}\left(x, u_{0}\right)\right)_{v} \\
=\varphi_{z}\left(x, u_{0}\right)\left(\operatorname{det}^{1 / n}\left(D_{x}^{2} u_{0}\right)-g_{1}\left(x, u_{0}\right)\right) .
\end{gathered}
$$

Elliptic equations of Monge-Ampère type have been explored in [7-10] by using the continuity method. Some of the techniques used there will be applied in our paper as well. For the parabolic case, Schnürer and Smoczyk [6] consider the flow of a strictly convex hypersurface driven by the Gauss curvature. For the Neumann boundary value problem and for the second boundary value problem, they show that such a flow exists for all times and converges eventually to a solution of the prescribed Gauss curvature equation. Zhou and Lian [11] proved the existence and uniqueness of classical solutions to the third initial and boundary value problem for equation of parabolic Monge-Ampère type of the form $-\partial u / \partial t+\operatorname{det}^{1 / n}\left(D_{x}^{2} u\right)=f(x, t)$. In this paper we will consider more general case than [11] under the structure and compatibility conditions analogous to [6] and extend some results in [7] from elliptic case to parabolic case.

The organization of this paper is as follows. In Section 2, we will review some notations, definitions, and results. In Section 3, we will obtain the uniqueness of the strictly convex classical solutions by the comparison principle. In Section 4, we shall prove uniform estimates for $|\dot{u}|$. This will be used in Section 5 to derive $C^{0}$-estimates. $C^{1}$-estimates then follow from [7]. In Section 6, we shall derive $C^{2}$-estimates and the $C^{2+\beta, 1+\beta / 2}$-estimates. In Section 7 , we will give the proof of Theorem 1 .

Our main result is as follows.

Theorem 1 (the main theorem). Assume that $\Omega$ is a bounded, uniformly convex domain in $R^{n}$ with the boundary $\partial \Omega \in$ $C^{4+\alpha} . v$ denotes the unit inner normal on $\partial \Omega$ which has been extended on $\overline{Q_{T}}$ to become a properly smooth vector field independent of $t$. Let $g_{\sigma} \in C^{2+\alpha, 2+\alpha}(\bar{\Omega} \times R), \sigma=1,2$, and $\varphi \in C^{3+\alpha, 3+\alpha}(\bar{\Omega} \times R)$ that satisfy (2)-(3). Let $u_{0} \in C^{4+\alpha}(\bar{\Omega})$ be a strictly convex function that satisfies (4). Moreover, the compatibility conditions (5) are fulfilled. Then there exists a unique strictly convex solution of (1) in $K^{4+\alpha}$ for some $\alpha \epsilon$ $(0,1)$, where

$$
\begin{gathered}
K^{4+\alpha}:=\left\{v(x, t) \mid v(x, t) \in C^{2,1}\left(Q_{T}\right) \cap C^{1,0}\left(\overline{Q_{T}}\right)\right. \\
\text { and } v(\cdot, t) \text { is strictly convex for every time } \\
t \in[0, T]\} \cap C^{4+\alpha, 2+\alpha / 2}\left(\overline{Q_{T}}\right), \\
Q_{T}=\Omega \times(0, T] .
\end{gathered}
$$

As $t \rightarrow \infty$, the functions $\left.u\right|_{t}$ converge to a limit function $u^{\infty}$ such that $u^{\infty}$ is of class $C^{4}(\bar{\Omega})$ and satisfies the Neumann boundary value problem

$$
\begin{gathered}
\operatorname{det}^{1 / n}\left(D_{x}^{2} u^{\infty}\right)=g_{2}\left(x, u^{\infty}\right) \quad \text { in } \Omega \\
u_{v}^{\infty}(x)=\varphi\left(x, u^{\infty}\right) \quad \text { on } \partial \Omega,
\end{gathered}
$$

where $v$ is the inward ponting unit normal of $\partial \Omega$.
Proof. Uniqueness of the strictly convex classical solution is given by Theorem 5. From the estimates obtained in Sections $4-6$, we get the existence and the asymptotic behavior of the classical solution in Section 7.

\section{Review of Some Notations, Definitions, and Results}

We first review some notations and definitions as follows:

$R^{n}$ is the $n$-dimensional Euclidean space with $n \geq 2$;

$\Omega$ is a bounded, uniformly convex domain in $R^{n}$, and $\partial \Omega$ denotes the boundary of $\Omega$;

$Q_{T}=\Omega \times(0, T]$, and $\partial_{P} Q_{T}$ denotes the parabolic boundary of $Q_{T}, \partial_{P} Q_{T}=\overline{Q_{T}}-Q_{T}$;

$\dot{u}=\partial u / \partial t, \ddot{u}=\partial^{2} u / \partial t^{2}$;

$u_{i}=D_{i} u=\partial u / \partial x_{i}, D u=\left(D_{1} u, \ldots, D_{n} u\right) ;$

$|D u|^{2}:=\sum_{i=1}^{n}\left|D_{i} u\right|^{2}, D_{i j}:=\partial^{2} / \partial x_{i} x_{j} ;$

$\left(u^{i j}\right)$ denotes the inverse of $\left(u_{i j}\right)$;

$\operatorname{tr}\left(D_{x}^{2} u\right)$ denotes the trace of the Hessian matrix $\left(u_{i j}\right)$; $\operatorname{det}\left(D_{x}^{2} u\right)$ denotes the determinant of the Hessian matrix $\left(u_{i j}\right)$;

$C^{l, k}\left(Q_{T}\right):=\left\{u(x, t) \mid D_{x}^{i} u\right.$ and $D_{t}^{j} u$ are all continuous in $\left.Q_{T}(0 \leq i \leq l, 0 \leq j \leq k)\right\}$;

$C^{2 l+\alpha, l+\alpha / 2}:=\left\{u ; D^{\beta} D_{t}^{r} u \in C^{\alpha, \alpha / 2}\left(\overline{Q_{T}}\right)\right.$, for all $\beta$ and $r$ that satisfy $|\beta|+2 r \leq 2 l\}$ with the norm

$$
|u|_{2 l+\alpha, l+\alpha / 2 ; \mathrm{Q}_{T}}=\sum_{|\beta|+2 r \leq 2 l}\left|D^{\beta} D_{t}^{r} u\right|_{\alpha, \alpha / 2 ; \mathrm{Q}_{T}} .
$$

Indices $z$ and $p_{i}$ denote partial derivatives with respect to the argument used for the function $u$ and for its gradient, respectively. This paper adopts the Einstein summation convention and sums over repeated Latin indices from 1 to $n$. For example, $u_{i} v^{i}$ means $\sum_{i=1}^{n} u_{i} v^{i}$. We will say "a constant $C$ under control" or "a controllable constant $C$ " if the constant $C$ (independent of $T$ ) depends only on the known or estimated quantities, for example, the $C^{4}$ normal of $u_{0}$ and $n$-the dimension of $R^{n}$. We point out that the inequalities remain valid when $C$ is enlarged.

Now, we state existence results.

Lemma 2 (see [11]). Let $F\left(D_{x}^{2} u\right)=\operatorname{det}^{1 / n}\left(D_{x}^{2} u\right), F^{i j}\left(D_{x}^{2} u\right)=$ $\partial F\left(D_{x}^{2} u\right) / \partial u_{i j}$; then $F\left(D_{x}^{2} u\right)$ is a concave function, $\left(F^{i j}\left(D_{x}^{2} u\right)\right)$ is a positive matrix, and $\operatorname{tr}\left(F^{i j}\left(D_{x}^{2} u\right)\right)=\sum_{i=1}^{n} F^{i i}\left(D_{x}^{2} u\right) \geq 1$.

Lemma 3 (see [12]). If $f \in C^{2}([0,1])$, then there exists a constant $M$ which is independent of $f$, such that

$$
\left\|f^{\prime}\right\| \leq M\|f\|\left(\|f\|+\left\|f^{\prime \prime}\right\|\right)
$$

where $\|f\|=\max \{|f(x)|: 0 \leq x \leq 1\}$. 


\section{Comparison Principle and Uniqueness}

This section is concerned with the uniqueness of the strictly convex classical solution for (1). First of all, we will prove a comparison principle as follows.

Lemma 4. Assume that $u, v \in C^{2,1}\left(\overline{Q_{T}}\right)$ and $u(\cdot, t), v(\cdot, t)$ are all convex for every time $t \in(0, T]$. For some $T_{0}, T_{0} \in(0, T)$, when $t \in\left(0, T_{0}\right], g_{\sigma}(x, u)=g_{1}(x, u)$, and when $t \in\left(T_{0}, T\right]$, $g_{\sigma}(x, u)=g_{2}(x, u)$. Let $g_{\sigma} \in C^{2,2}(\bar{\Omega} \times R), \sigma=1,2$, and $\left(g_{\sigma}\right)_{z}=$ $\partial g_{\sigma}(x, z) / \partial z \geq 0$. Moreover, assume that

$$
\begin{aligned}
& \text { (1) }-\dot{u}+\operatorname{det}^{1 / n}\left(D_{x}^{2} u\right)-g_{\sigma}(x, u) \geq-\dot{v}+\operatorname{det}^{1 / n}\left(D_{x}^{2} v\right)- \\
& g_{\sigma}(x, v) \text { in } \Omega \times(0, T], \\
& \text { (2) if } u>v \text {, then } u_{v}>v_{v} \text { on } \partial \Omega \times[0, T], \\
& \text { (3) } u \leq v \text { on } \Omega \times\{t=0\},
\end{aligned}
$$

where $v$ is the inward pointing unit normal of $\partial \Omega$; then $u \leq v$ in $\overline{Q_{T}}$.

Proof. Consider

$$
\begin{aligned}
-\dot{u}+\operatorname{det}^{1 / n}\left(D_{x}^{2} u\right)-g_{\sigma}(x, u)-\left(-\dot{v}+\operatorname{det}^{1 / n}\left(D_{x}^{2} v\right)-g_{\sigma}(x, v)\right) \\
=-(\dot{u}-\dot{v}) \\
\quad+\int_{0}^{1} \frac{\partial \operatorname{det}^{1 / n}\left[s D_{x}^{2} u+(1-s) D_{x}^{2} v\right]}{\partial\left(s u_{i j}+(1-s) v_{i j}\right)} d s(u-v)_{i j} \\
\quad-\int_{0}^{1} \frac{\partial g_{\sigma}(x, s u+(1-s) v)}{\partial(s u+(1-s) v)} d s(u-v) \\
=-(\dot{u}-\dot{v})+a^{i j}(u-v)_{i j}-b(u-v),
\end{aligned}
$$

where $a^{i j}=\int_{0}^{1}\left(\partial \operatorname{det}^{1 / n}\left[s D_{x}^{2} u+(1-s) D_{x}^{2} v\right] / \partial\left(s u_{i j}+(1-\right.\right.$ s) $\left.\left.v_{i j}\right)\right) d s, b=\int_{0}^{1}\left(\partial g_{\sigma}(x, s u+(1-s) v) / \partial(s u+(1-s) v)\right) d s$.

From the assumptions and Lemma 2 , we obtain that $\left(a^{i j}\right)$ is a positive matrix and $b \geq 0$.

Combining (10) with condition (1), we infer that

$$
-(\dot{u}-\dot{v})+a^{i j}(u-v)_{i j}-b(u-v) \geq 0 \quad \text { in } \Omega \times(0, T] ;
$$

an application of the weak parabolic maximum principle gives $\max _{\overline{Q_{T}}}(u-v) \leq \max _{\partial_{P} Q_{T}}(u-v)^{+}$. In addition, from condition (2), $u-v$ cannot admit a positive maximum on $\partial \Omega \times[0, T]$. And from condition (3), $u \leq v$ on $\Omega \times\{t=0\}$. So we obtain that $u \leq v$ in $\overline{Q_{T}}$.

Theorem 5. Under the assumptions of Theorem 1, there exists a unique classical solution of (1).

Proof. Assume that $u, v \in C^{2,1}\left(\overline{Q_{T}}\right)$ are two classical solutions of (1). Then we have

$$
\begin{gathered}
\dot{u}=\operatorname{det}^{1 / n}\left(D_{x}^{2} u\right)-g_{\sigma}(x, u) \quad \text { in } \Omega \times(0, T], \\
u=u_{0}(x) \quad \text { on } \Omega \times\{t=0\} ;
\end{gathered}
$$

meanwhile,

$$
\begin{gathered}
\dot{v}=\operatorname{det}^{1 / n}\left(D_{x}^{2} v\right)-g_{\sigma}(x, v) \quad \text { in } \Omega \times(0, T], \\
v=u_{0}(x) \quad \text { on } \Omega \times\{t=0\} .
\end{gathered}
$$

Thus,

$$
\begin{gathered}
-\dot{u}+\operatorname{det}^{1 / n}\left(D_{x}^{2} u\right)-g_{\sigma}(x, u) \\
=-\dot{v}+\operatorname{det}^{1 / n}\left(D_{x}^{2} v\right)-g_{\sigma}(x, v) \quad \text { in } \Omega \times(0, T], \\
u=v \quad \text { on } \Omega \times\{t=0\} .
\end{gathered}
$$

It follows that conditions (1) and (3) in Lemma 4 are satisfied.

From $u_{v}=\varphi(x, u)$ on $\partial \Omega \times[0, T]$ and the structure condition (2), we obtain that condition (2) in Lemma 4 is satisfied.

Since $g_{\sigma} \in C^{2+\alpha, 2+\alpha}(\bar{\Omega} \times R)$ and the structure condition (3) is satisfied, we obtained from Lemma 4 that $u=v$ for all $(x, t) \in \overline{Q_{T}}$.

\section{4. $\dot{u}$-Estimates}

The proof of the $\dot{u}$-estimates can be carried out as in [6]. For a constant $\lambda$ we define the function $r=e^{\lambda t}(\dot{u})^{2}$; thus

$$
\begin{aligned}
\dot{r}= & \lambda e^{\lambda t}(\dot{u})^{2}+2 e^{\lambda t} \dot{u} \ddot{u} \\
= & \lambda r+2 e^{\lambda t} \dot{u} \frac{d}{d t}\left(\operatorname{det}^{1 / n}\left(D_{x}^{2} u\right)-g_{\sigma}(x, u)\right) \\
= & \lambda r+\frac{2}{n} e^{\lambda t} \dot{u} \dot{u}_{i j} u^{i j} \cdot \operatorname{det}^{1 / n}\left(D_{x}^{2} u\right)-2 r\left(g_{\sigma}\right)_{z} \\
= & \lambda r+\frac{1}{n}\left(r_{i j}-2 e^{\lambda t} \dot{u}_{i} \dot{u}_{j}\right) u^{i j} \cdot \operatorname{det}^{1 / n}\left(D_{x}^{2} u\right)-2 r\left(g_{\sigma}\right)_{z} \\
= & \frac{1}{n} \operatorname{det}^{1 / n}\left(D_{x}^{2} u\right) u^{i j} r_{i j}-\frac{2}{n} e^{\lambda t} \operatorname{det}^{1 / n}\left(D_{x}^{2} u\right) u^{i j} \dot{u}_{i} \dot{u}_{j} \\
& +\left(\lambda-2\left(g_{\sigma}\right)_{z}\right) r .
\end{aligned}
$$

So (1) implies the following evolution equation for $r$ :

$$
\begin{aligned}
\dot{r}= & \frac{1}{n} \operatorname{det}^{1 / n}\left(D_{x}^{2} u\right) u^{i j} r_{i j}-\frac{2}{n} e^{\lambda t} \operatorname{det}^{1 / n}\left(D_{x}^{2} u\right) u^{i j} \dot{u}_{i} \dot{u}_{j} \\
& +\left(\lambda-2\left(g_{\sigma}\right)_{z}\right) r .
\end{aligned}
$$

Theorem 6. As long as a strictly convex solution of (1) exists, one obtains the estimates

$$
|\dot{u}|_{0, \bar{Q}_{T}} \leq \bar{M},
$$

where $\bar{M}$ is a controllable constant.

Proof. If $(\dot{u})^{2}$ admits a positive local maximum in $x \in \partial \Omega$ for a positive time, then we differentiate the Neumann boundary condition and obtain from (2) that

$$
\left((\dot{u})^{2}\right)_{v}=2 \dot{u}(\dot{u})_{v}=2 \dot{u}\left(\dot{u}_{\nu}\right)=2(\dot{u})^{2} \varphi_{z}>0
$$

which contradicts the maximality of $(\dot{u})^{2}$ at $x$. 
Now we choose $\lambda=0$ in (16) and get

$$
\begin{aligned}
\frac{d(\dot{u})^{2}}{d t}= & \frac{1}{n} u^{i j} \operatorname{det}^{1 / n}\left(D_{x}^{2} u\right)\left((\dot{u})^{2}\right)_{i j} \\
& -\frac{2}{n} u^{i j} \operatorname{det}^{1 / n}\left(D_{x}^{2} u\right) \dot{u}_{i} \dot{u}_{i}-2\left(g_{\sigma}\right)_{z}(\dot{u})^{2} \\
\leq & \frac{1}{n} \operatorname{det}^{1 / n}\left(D_{x}^{2} u\right) u^{i j}\left((\dot{u})^{2}\right)_{i j}-2\left(g_{\sigma}\right)_{z}(\dot{u})^{2} .
\end{aligned}
$$

Since det $D_{x}^{2} u>0,\left(g_{\sigma}\right)_{z} \geq 0$, we obtain from the parabolic maximum principle that

$$
\max _{\overline{Q_{T}}}(\dot{u})^{2} \leq \max _{\partial_{P} Q_{T}}\left((\dot{u})^{2}\right)^{+} .
$$

From the aforementioned a positive local maximum of $(\dot{u})^{2}$ cannot occur at a point of $\partial \Omega$ for a positive time, so

$$
(\dot{u})^{2} \leq \max _{t=0}(\dot{u})^{2} \Longrightarrow|\dot{u}| \leq \max _{t=0}|\dot{u}|
$$

From the fact that the solution is smooth up to the initial time $t=0$, we get

$$
\dot{u}=\operatorname{det}^{1 / n}\left(D_{x}^{2} u_{0}\right)-g_{1}\left(x, u_{0}\right) \quad \text { on } \bar{\Omega} \times\{t=0\} .
$$

By (21) and (22), there exists a controllable constant $\bar{M}$ such that $|\dot{u}|_{0, \bar{Q}_{T}} \leq \bar{M}$. Here we have used the fact that $u_{0} \in C^{4}(\bar{\Omega})$.

Lemma 7. If $0 \leq \dot{u}(x, 0) \not \equiv 0$ for $t=0$, then a solution of (1) satisfies $\dot{u}>0$ or equivalently $\operatorname{det}^{1 / n}\left(D_{x}^{2} u\right)-g_{\sigma}(x, u)>0$ for $\sigma=1,2$ and $t>0$.

Proof. We use the methods known from [6]. Differentiating the equation

$$
\dot{u}=\operatorname{det}^{1 / n}\left(D_{x}^{2} u\right)-g_{\sigma}(x, u)
$$

yields

$$
\ddot{u}=\frac{1}{n} \operatorname{det}^{1 / n}\left(D_{x}^{2} u\right) u^{i j} \dot{u}_{i j}-\left(g_{\sigma}\right)_{z} \dot{u} .
$$

From (24) and parabolic maximum principle, we see that

$$
\inf _{Q_{T}}(\dot{u}) \geq \inf _{\partial_{P} Q_{T}}(\dot{u})_{-}
$$

where $(\dot{u})_{-}=\min \{\dot{u}, 0\}$.

If $\dot{u}$ admits a negative local minimum in $x \in \partial \Omega$ for a positive time, then we differentiate the Neumann boundary condition and get from (2) that

$$
(\dot{u})_{v}=\left(\dot{u}_{\nu}\right)=\varphi_{z} \dot{u}<0
$$

which contradicts the minimum of $(\dot{u})$ at $x$. Since $0 \leq \dot{u}(x, 0)$, it follows that $\inf _{\partial_{P} Q_{T}}(\dot{u})_{-}=0$. That is,

$$
\inf _{Q_{T}}(\dot{u}) \geq \inf _{\partial_{P} Q_{T}}(\dot{u})_{-}=0 .
$$

So $\dot{u} \geq 0$ or equivalently $\operatorname{det}^{1 / n}\left(D_{x}^{2} u\right)-g_{\sigma}(x, u) \geq 0$ for $\sigma=$ 1,2 and $t>0$.

From (24) and the strong parabolic maximum principle [13], we obtain that $\dot{u}$ has to vanish identically if it vanishes in $\left(x_{0}, t_{0}\right) \in \Omega \times(0, T]$, contradicting $\dot{u} \not \equiv 0$ for $t=0$. If $\dot{u}=0$ for $x_{0} \in \partial \Omega$, the Neumann boundary condition implies that

$$
(\dot{u})_{v}=(\dot{u}) \varphi_{z}=0,
$$

but this is impossible in view of the Hopf lemma applied to (24).

Consequently, if $0 \leq \dot{u}(x, 0) \not \equiv 0$ for $t=0$, then a solution of (1) satisfies $\dot{u}>0$ or equivalently $\operatorname{det}^{1 / n}\left(D_{x}^{2} u\right)-g_{\sigma}(x, u)>0$ for $\sigma=1,2$ and $t>0$.

\section{5. $C^{0}$ - and $C^{1}$-Estimates}

In this section we derive the $C^{0}$ - and $C^{1}$-estimates of the solution to problem (1).

Theorem 8. Let $\Omega$ be a bounded, uniformly convex domain in $R^{n}$. Also, $u \in C^{2,1}\left(Q_{T}\right) \cap C^{1,0}\left(\overline{Q_{T}}\right)$ is a strictly convex solution of (1). Then there exists a controllable constant $M_{0}$, such that $|u|_{0, \overline{Q_{T}}} \leq M_{0}$.

Proof. Since (4) is satisfied, we obtained from Lemma 7 that $\dot{u} \geq 0$ in $\overline{Q_{T}}$. So $u(x, t) \geq u(x, 0)=u_{0}(x)$. As $u_{0}(x) \in C^{4+\alpha}(\bar{\Omega})$, then there exists a controllable constant $N_{1}$ such that

$$
u(x, t) \geq N_{1} \quad \text { in } \overline{Q_{T}} .
$$

Next we will prove that $u$ is uniformly a priori bounded from above.

At a maximum of $u$, which necessarily occurs on $\partial \Omega$ since $u$ is convex, we have $u_{v} \leq 0$. Since $u_{v}=\varphi(x, u)$ on $\partial \Omega \times[0, T]$, then

$$
\varphi(x, u) \leq 0 \quad(x, t) \in \partial \Omega \times[0, T] .
$$

From (2) we get that $\varphi(\cdot, z) \rightarrow \infty$ uniformly as $z \rightarrow \infty$. Then we can deduce that $\varphi(x, z)>0$ for all $x \in \partial \Omega$ and $z>$ $N_{2}$, where $N_{2}$ is controllable constant. Combining (30) yields

$$
u \leq N_{2} \text {. }
$$

This completes the proof of the theorem.

Theorem 9. Let $\Omega$ be a bounded, uniformly convex domain in $R^{n}$, and $u \in C^{4,2}\left(Q_{T}\right) \cap C^{1,0}\left(\overline{Q_{T}}\right)$ is a strictly convex solution of (1). Then one has

$$
\sup _{\mathrm{Q}_{T}}|D u| \leq M^{*}
$$

where $M^{*}$ is a controllable constant.

Proof. For any $t_{0} \in[0, T], u\left(x, t_{0}\right)$ is a continuous differentiable, convex function. From $u_{v}=\varphi(x, u)$ and the $C^{0}$ estimates, we get

$$
u_{v} \geq-M
$$


where $M$ is a controllable constant. Then using Theorem 2.2 in [7], we have

$$
\left|D u\left(x, t_{0}\right)\right| \leq M_{1} \text { on } \bar{\Omega} .
$$

Since $t_{0}$ is arbitrary, we obtain that

$$
|D u| \leq M^{*}
$$

where $M^{*}$ is a controllable constant. This completes the proof of the theorem.

\section{6. $C^{2}$ - and $C^{2+\beta, 1+\beta / 2}$-Estimates}

This section is concerned with the $C^{2}$-estimates and the $C^{2+\beta, 1+\beta / 2}$-estimates of the solution to problem (1).

Theorem 10. Assume that $\Omega$ is a $C^{4}$ bounded, uniformly convex domain in $R^{n}$ and $u \in C^{4,2}\left(\overline{Q_{T}}\right)$ is a strictly convex solution of (1). Let $g_{\sigma} \in C^{2,2}(\bar{\Omega} \times R), \sigma=1,2, \varphi \in C^{3,3}(\bar{\Omega} \times R)$. Then one has

$$
\sup _{\mathrm{Q}_{T}}\left|D_{x}^{2} u\right| \leq M^{\prime \prime}
$$

where $M^{\prime \prime}$ is a controllable constant.

Proof. Let $\xi \in S^{n-1}$. First we observe that $D_{\xi \xi} u>0$, since $u$ is strictly convex. So we only need to prove the fact that $D_{\xi \xi} u$ is a priori bounded from above.

We define for $(x, t, \xi) \in \bar{\Omega} \times[0, T] \times S^{n-1}$ that

$$
\omega(x, t, \xi)=D_{\xi \xi} u-V(x, t, \xi)+K|x|^{2},
$$

where $V(x, \xi, t)$ is given by

$$
V(x, \xi, t)=2\langle\xi, \nu\rangle \xi_{i}^{\prime}\left(D_{i} \varphi-D_{k} u D_{i} \nu_{k}\right) .
$$

Here $v$ is a smooth extension of the inner unit normal on $\partial \Omega$ that is independent of $t$. $\xi^{\prime}$ is given by

$$
\xi^{\prime}=\xi-\langle\xi, \nu\rangle \nu
$$

$K$ is a constant to be chosen, and $D$ indicates that the chain rule has not yet been applied to the respective terms.

Let

$$
\begin{gathered}
a_{k}=2\langle\xi, \nu\rangle\left(\varphi_{z} \xi_{k}^{\prime}-\xi_{i}^{\prime} D_{i} \nu_{k}\right), \\
b=2\langle\xi, \nu\rangle \xi_{i}^{\prime} \varphi_{i},
\end{gathered}
$$

then

$$
\begin{gathered}
V(x, \xi, t)=a_{k} u_{k}+b, \\
\omega(x, t, \xi)=D_{\xi \xi} u-a_{k} u_{k}-b+K|x|^{2} .
\end{gathered}
$$

We compute that

$$
\begin{aligned}
-\frac{\partial \omega}{\partial t}+F^{i j} D_{i j} \omega= & -D_{\xi \xi t} u+a_{k} D_{t k} u+D_{t} a_{k} \cdot u_{k}+D_{t} b \\
& +F^{i j} D_{i j \xi \xi} u-a_{k} F^{i j} \cdot u_{i j k}-2 F^{i j} D_{i} a_{k} \cdot u_{k j} \\
& -F^{i j} \cdot u_{k} D_{i j} a_{k}-F^{i j} D_{i j} b+2 K F^{i j} \delta_{i j} .
\end{aligned}
$$

Next, we estimate the right-hand side of (42), respectively.

Let $F\left(D_{x}^{2} u\right)=\operatorname{det}^{1 / n}\left(D_{x}^{2} u\right)$. From Lemma 2, we have that $F\left(D_{x}^{2} u\right)$ is a concave function, $\left(F^{i j}\left(D_{x}^{2} u\right)\right)$ is a positive matrix, and $\operatorname{tr}\left(F^{i j}\left(D_{x}^{2} u\right)\right)=\sum_{i=1}^{n} F^{i i}\left(D_{x}^{2} u\right) \geq 1$.

Differentiating the equation

$$
\dot{u}=F\left(D_{x}^{2} u\right)-g_{\sigma}(x, u)
$$

twice in the direction $\xi, \xi \in S^{n-1}$, we therefore obtain

$$
-D_{t \xi \xi} u+F^{i j} D_{i j \xi \xi} u+F^{i j, k l} D_{i j \xi} u D_{k l \xi} u=D_{\xi \xi} g_{\sigma}(x, u) \text {. }
$$

Using the concavity of $F$, we have

$$
F^{i j, k l} D_{i j \xi} u D_{k l \xi} u \leq 0 ;
$$

then

$$
-D_{t \xi \xi} u+F^{i j} D_{i j \xi \xi} u \geq D_{\xi \xi} g_{\sigma}(x, u) .
$$

Differentiating the equation

$$
\dot{u}=F\left(D_{x}^{2} u\right)-g_{\sigma}(x, u)
$$

in the $k$ th coordinate direction, we obtain

$$
-D_{t k} u+F^{i j} D_{i j k} u=D_{k} g_{\sigma} .
$$

From $\left(F^{i j}\right)=\partial F\left(D_{x}^{2} u\right) / \partial u_{i j}=\left(F\left(D_{x}^{2} u\right) / n\right) u^{i j}$, where $\left(u^{i j}\right)$ is the inverse of $\left(u_{i j}\right)$, we have

$$
F^{i j} D_{i} a_{k} \cdot u_{k j}=\frac{F\left(D_{x}^{2} u\right)}{n} u^{i j} u_{k j} \cdot D_{i} a_{k}=\frac{F\left(D_{x}^{2} u\right)}{n} D_{i} a_{i}
$$

Using the estimates of $\dot{u}$ and $u$, we obtain that $F\left(D_{x}^{2} u\right)=\dot{u}+g_{\sigma}$ is bounded. From

$$
\begin{aligned}
D_{j} a_{j}= & 2 \xi_{l} D_{j} \nu_{l}\left(\varphi_{z} \xi_{j}^{\prime}-\xi_{i}^{\prime} D_{i} \nu_{j}\right) \\
& +2\langle\xi, v\rangle\left[\left(\varphi_{z j}+\varphi_{z z} D_{j} u\right) \xi_{j}^{\prime}-\xi_{i}^{\prime} D_{j i} \nu_{j}\right],
\end{aligned}
$$

as well as $C^{0}$ - and $C^{1}$-estimates, it follows that $\left|D_{j} a_{j}\right|$ is bounded. Thus there exists a controllable constant $C$ such that

$$
\left|F^{i j} D_{i} a_{k} \cdot u_{k j}\right| \leq C
$$

Since $\left(F^{i j}\right)$ is positive definite, we can get that

$$
\left|F^{i j}\right| \leq \frac{1}{2}\left(F^{i i}+F^{j j}\right)
$$

Applying (52), $C^{1}$-estimates, and the following equality:

$$
\begin{aligned}
D_{j h} a_{k}= & 2 \xi_{l} D_{j h} \nu_{l}\left(\varphi_{z} \xi_{k}^{\prime}-\xi_{i}^{\prime} D_{i} \nu_{k}\right) \\
& +2 \xi_{l} D_{j} \nu_{l}\left[\left(\varphi_{z h}+\varphi_{z z} u_{h}\right) \xi_{k}^{\prime}-\xi_{i}^{\prime} D_{i h} \nu_{k}\right] \\
& +2 \xi_{s} D_{h} \nu_{s}\left[\left(\varphi_{z j}+\varphi_{z z} u_{j}\right) \xi_{k}^{\prime}-\xi_{i}^{\prime} D_{i j} \nu_{k}\right] \\
& +2\langle\xi, \nu\rangle\left\{\left[\left(\varphi_{z j h}+\varphi_{z j z} u_{h}\right)+\left(\varphi_{z z h}+\varphi_{z z z} u_{h}\right) u_{j}\right.\right. \\
& \left.\left.+\varphi_{z z} u_{j h}\right] \xi_{k}^{\prime}-\xi_{i}^{\prime} D_{i j h} \nu_{k}\right\},
\end{aligned}
$$


we obtain that

$$
\begin{aligned}
\left|F^{h j} \cdot u_{k} D_{h j} a_{k}\right| & \leq\left|u_{k} F^{h j} 2\langle\xi, \nu\rangle \varphi_{z z} u_{j h} \xi_{k}^{\prime}\right|+C\left|F^{h j}\right| \\
& =\left|2 u_{k}\langle\xi, v\rangle \varphi_{z z} \xi_{k}^{\prime} \frac{F\left(D_{x}^{2} u\right)}{n} u^{h j} u_{j h}\right|+C\left|F^{h j}\right| \\
& \leq C_{1}+C_{2} \operatorname{tr}\left(F^{i j}\right)
\end{aligned}
$$

where $C_{1}$ and $C_{2}$ are positive controllable constants.

From (51), (54), and the estimates like these, it follows that

$$
\begin{aligned}
& \left|D_{t} a_{k} \cdot u_{k}+D_{t} b-2 F^{i j} D_{i} a_{k} \cdot u_{k j}-F^{i j} \cdot u_{k} D_{i j} a_{k}-F^{i j} D_{i j} b\right| \\
& \quad \leq c_{1} \operatorname{tr}\left(F^{i j}\right)+c_{2}
\end{aligned}
$$

where $c_{1}$ and $c_{2}$ are positive controllable constants. Then using (46) and (48), we can obtain

$$
\begin{aligned}
-\frac{\partial \omega}{\partial t}+F^{i j} D_{i j} \omega \geq & D_{\xi \xi} g_{\sigma}-a_{k} D_{k} g_{\sigma} \\
& -\left(c_{1} \operatorname{tr}\left(F^{i j}\right)+c_{2}\right)+2 K F^{i j} \delta_{i j} \\
= & \left(g_{\sigma}\right)_{\xi \xi}+2\left(g_{\sigma}\right)_{\xi z} u_{\xi}+\left(g_{\sigma}\right)_{z z} u_{\xi} u_{\xi} \\
& +\left(g_{\sigma}\right)_{z} u_{\xi \xi}-a_{k}\left(\left(g_{\sigma}\right)_{k}+\left(g_{\sigma}\right)_{z} u_{k}\right) \\
& -\left(c_{1} \operatorname{tr}\left(F^{i j}\right)+c_{2}\right)+2 K F^{i j} \delta_{i j} \\
\geq & \left(g_{\sigma}\right)_{\xi \xi}+2\left(g_{\sigma}\right)_{\xi z} u_{\xi}+\left(g_{\sigma}\right)_{z z} u_{\xi} u_{\xi} \\
& -a_{k}\left(\left(g_{\sigma}\right)_{k}+\left(g_{\sigma}\right)_{z} u_{k}\right) \\
& -\left(c_{1} \operatorname{tr}\left(F^{i j}\right)+c_{2}\right)+2 K F^{i j} \delta_{i j},
\end{aligned}
$$

where we have used the structure condition (3) and the convexity of $u$. Using $C^{0}$ - and $C^{1}$-estimates, there exist positive controllable constants $c_{3}$ and $c_{4}$ such that

$$
-\frac{\partial \omega}{\partial t}+F^{i j} D_{i j} \omega \geq\left(2 K-c_{3}\right) \operatorname{tr}\left(F^{i j}\right)-c_{4} .
$$

Since $\operatorname{tr}\left(F^{i j}\right) \geq 1$, we fix $K \geq(1 / 2)\left(c_{3}+c_{4}+1\right)$ and deduce that

$$
-\frac{\partial \omega}{\partial t}+F^{i j} D_{i j} \omega \geq 1 .
$$

Thus by the parabolic maximum principle, we have

$$
\omega \leq \sup _{\partial_{P} Q_{T}} \omega
$$

As $\omega$ is known on $\bar{\Omega} \times\{t=0\} \times S^{n-1}$, we need only to estimate $\omega$ on $\partial \Omega \times[0, T] \times S^{n-1}$.
The estimation of $\omega$ on $\partial \Omega \times[0, T] \times S^{n-1}$ splits into four stages according to the direction $\xi$. The first three stages: (i) the mixed tangential normal second derivatives of $u$ on $\partial \Omega \times[0, T] \times S^{n-1}$, (ii) $\xi$ tangential, and (iii) $\xi$ nontangential, can be carried out as in [7]. The details of this procedure could be seen in [7]. Stage (i) is readily estimated. Stages (ii) and (iii) are reduced to the purely normal case. So we only give the proof of the fourth stage: (iv) $\xi$ normal. We extend the argument given in [2] and modified for the parabolic case.

Set $h(x, t)=v_{k} D_{k} u-\varphi(x, u)=D_{v} u-\varphi(x, u)$. By (48), a direct calculation yields

$$
\begin{aligned}
L h= & -D_{t} h+F^{i j} D_{i j} h \\
= & -v_{k} D_{k t} u+\varphi_{z} D_{t} u+v_{k} F^{i j} D_{i j k} u \\
& +2 F^{i j} D_{i} v_{k} D_{j k} u+F^{i j} D_{i j} v_{k} D_{k} u-F^{i j} D_{i j} \varphi \\
= & v_{k}\left(\left(g_{\sigma}\right)_{k}+\left(g_{\sigma}\right)_{z} u_{k}\right)+\varphi_{z} D_{t} u \\
& +2 F^{i j} D_{i} \nu_{k} D_{j k} u+F^{i j} D_{i j} \nu_{k} D_{k} u \\
& -F^{i j}\left(\varphi_{i j}+2 \varphi_{i z} u_{j}+\varphi_{z z} u_{i} u_{j}+\varphi_{z} u_{i j}\right) .
\end{aligned}
$$

Thus, using $\left(F^{i j}\right)=\left(F\left(D_{x}^{2} u\right) / n\right) u^{i j},(52)$, and our a priori estimates, we have

$$
|L h|=\left|-D_{t} h+F^{i j} D_{i j} h\right| \leq C_{0}\left(1+\operatorname{tr}\left(F^{i j}\right)\right) \leq C \operatorname{tr}\left(F^{i j}\right),
$$

where $C$ is a controllable constant.

Let $\left(x_{0}, t_{0}\right) \in \partial \Omega \times[0, T]$, and $\left(x_{0}, t_{0}\right)$ is arbitrary. We observe that $\Omega$ is a bounded, uniformly convex domain in $R^{n}$, so there exists a uniformly closed ball $B_{R}\left(x^{*}\right)$ such that

$$
\begin{gathered}
\Omega \subset B_{R}\left(x^{*}\right) \subset R^{n}, \\
\partial B_{R}\left(x^{*}\right) \cap \partial \Omega=\left\{x_{0}\right\} .
\end{gathered}
$$

Meanwhile, we assume that $\left|x-x^{*}\right|>1$ for all $x \in \bar{\Omega}$.

We consider the auxiliary function in $B_{R}\left(x^{*}\right) \times[0, T]$

$$
q(x, t)=e^{K_{1} R^{2}}-e^{K_{1}\left|x-x^{*}\right|^{2}}
$$

where $K_{1}$ is a positive constant to be determined.

If we choose $K_{1}$ sufficiently large, it is easy to see that $q(x, t) \geq h(x, t)$ on $\partial_{P} Q_{T}$. For sufficiently large $K_{1}$, we have

$$
\begin{aligned}
-\frac{\partial q}{\partial t}+F^{i j} D_{i j} q= & -2 K_{1} \operatorname{tr}\left(F^{i j}\right) e^{K_{1}\left|x-x^{*}\right|^{2}} \\
& -4 K_{1}^{2} F^{i j}\left(x-x^{*}\right)_{i}\left(x-x^{*}\right)_{j} e^{K_{1}\left|x-x^{*}\right|^{2}} \\
\leq & -2 K_{1} \operatorname{tr}\left(F^{i j}\right) e^{K_{1}\left|x-x^{*}\right|^{2}} \\
\leq & -C \operatorname{tr}\left(F^{i j}\right)
\end{aligned}
$$

where we have used the fact that $\left(F^{i j}\right)$ is positive definite. 
By (61) and (64), we get

$$
-\frac{\partial(q-h)}{\partial t}+F^{i j} D_{i j}(q-h) \leq 0 ;
$$

thus we obtain $q-h \geq \inf _{\partial_{P} Q_{T}}(q-h) \geq 0$ on $\overline{Q_{T}}$ in view of the parabolic maximum principle. Since $q\left(x_{0}, t_{0}\right)=h\left(x_{0}, t_{0}\right)=0$, it follows that

$$
\frac{(q-h)\left(x_{0}+\rho v\right)-(q-h)\left(x_{0}, t_{0}\right)}{\rho} \geq 0
$$

thus

$$
\lim _{\rho \rightarrow 0^{+}} \frac{(q-h)\left(x_{0}+\rho \nu\right)-(q-h)\left(x_{0}, t_{0}\right)}{\rho} \geq 0 .
$$

Therefore,

$$
D_{\nu}(q-h)\left(x_{0}, t_{0}\right) \geq 0 .
$$

Hence,

$$
h_{v}\left(x_{0}, t_{0}\right) \leq q_{v}\left(x_{0}, t_{0}\right) \leq c_{1},
$$

where $c_{1}$ is a controllable constant.

For $-q$, in a similar fashion we can obtain

$$
h_{\nu}\left(x_{0}, t_{0}\right) \geq-c_{2},
$$

where $c_{2}$ is a controllable constant.

Since $\left(x_{0}, t_{0}\right) \in \partial \Omega \times[0, T]$ is arbitrary, we obtain

$$
\sup _{\partial \Omega \times[0, T]} D_{\nu \nu} u \leq C,
$$

where $C$ is a controllable constant.

Combining the estimates of the four stages, we obtain that there exists a controllable constant $C$ such that $D_{\xi \xi} u \leq C$ on $\overline{Q_{T}}$.

Since $\xi$ is an arbitrary direction in $S^{n-1}$, now let $\xi=$ $e_{i} \pm e_{j} / 2^{1 / 2}$, where $e_{i}=(0,0 \ldots, 1, \ldots 0)=i$ th standard coordinate vector. Thus we can get the required bounded for $D_{x}^{2} u$ immediately. This completes the proof of the theorem.

From the uniform $C^{0}$-estimates, $\dot{u}$-estimates, and the assumptions on $g_{\sigma}, \sigma=0,1$, we can conclude that $F\left(D^{2} u\right)$ has a priori positive bound from below. And using the uniform $C^{2}$-estimates for $u$, we obtain that (1) is uniformly parabolic. So we can apply the method of [14] to obtain the $C^{2+\beta, 1+\beta / 2}$ interior estimates and the estimates near the bottom. Using the estimates near the side in [15], we can get the Hölder seminorm estimates for $\dot{u}$ and $D_{x}^{2} u$. Thus we have the $C^{2+\beta, 1+\beta / 2}$-estimates.

\section{The Proof of Theorem 1}

In Section 3 we proved the uniqueness of the strictly convex solution for (1). The existence of the strictly convex solution for (1) is obtained by using the continuity method. Applying
Theorem 5.3 in [16], the implicit function theorem, and the Arzela-Ascoli theorem, we can get the desired result. Then the standard regularity of parabolic equation implies that $u \in$ $C^{4+\beta, 2+\beta / 2}\left(\overline{Q_{T}}\right)$. Since there are sufficient a priori estimates, we can extend a solution of (1) on a time interval $[0, T]$ to $[0, T+\epsilon)$ for a small $\epsilon>0$. In this way we obtain existence for all $t \geq 0$ from the a priori estimates. We then need the following lemma to prove the asymptotic behavior of a classical solution of (1).

Lemma 11. If a solution of (1) exists for all $t \geq 0$ and (4) is satisfied, then as $t \rightarrow \infty$, the functions $\left.u\right|_{t}$ converge to a limit function $u^{\infty}(x)$ such that $u^{\infty}(x)$ satisfies the Neumann boundary value problem

$$
\begin{gathered}
\operatorname{det}^{1 / n}\left(D_{x}^{2} u^{\infty}\right)=g_{2}\left(x, u^{\infty}\right) \quad x \in \Omega, \\
u_{v}^{\infty}=\varphi\left(x, u^{\infty}\right) \quad x \in \partial \Omega,
\end{gathered}
$$

where $v$ is the unit inner normal on $\partial \Omega$. Moreover, $u(x, t) \rightarrow$ $u^{\infty}(x)$ in $C^{3}$-norm.

Proof. We may assume that $\dot{u}(\cdot, 0) \neq \equiv 0$ and proceed as in [17]. Integrating the equation

$$
\dot{u}=\operatorname{det}^{1 / n}\left(D_{x}^{2} u\right)-g_{2}(x, u)
$$

with respect to $t$ yields

$$
\begin{aligned}
& u(x, t)-u\left(x, T_{0}\right) \\
& \quad=\int_{T_{0}}^{t}\left(\operatorname{det}^{1 / n}\left(D_{x}^{2} u(x, \tau)\right)-g_{2}(x, u(x, \tau))\right) d \tau .
\end{aligned}
$$

The left-hand side is uniformly bounded in view of the $C^{0}$-estimates. By applying Lemma $7, \operatorname{det}^{1 / n}\left(D_{x}^{2} u\right)-g_{2}$ is nonnegative, and we can find that $t_{k}=t_{k}(x) \rightarrow \infty$ such that

$$
\left.\left(\operatorname{det}^{1 / n}\left(D_{x}^{2} u\right)-g_{2}\right)\right|_{t=t_{k}} \longrightarrow 0 .
$$

On the other hand, $u(x, \cdot)$ is monotone, and therefore

$$
\lim _{t \rightarrow \infty} u(x, t)=: u^{\infty}(x)
$$

exists and is of class $C^{4}(\bar{\Omega})$ in view of the a priori estimates.

From differential interpolation inequality in Lemma 3, we can obtain the interpolation inequality of the form

$$
\|D \widetilde{u}\| \leq C\|\widetilde{u}\| \cdot\left(\left\|D_{x}^{2} \widetilde{u}\right\|+\|\widetilde{u}\|\right)
$$

for $\tilde{u}=u-u^{\infty}$, where $\|\cdot\|$ denotes the sup-norm.

Dini's theorem and interpolation inequalities of the form (77) yield $u(x, t) \rightarrow u^{\infty}(x)$ in $C^{3}$-norm. We finally, obtain in view of (75) that $u^{\infty}$ is a solution of the problem (72). This complete, the proof of the lemma.

Now we completed the proof of Theorem 1.

\section{Acknowledgment}

This project is supported by Inner Mongolia Natural Science Foundation of China under Grant 2011MS0107. 


\section{References}

[1] J.-F. Cossette and P. K. Smolarkiewicz, "A Monge-Ampère enhancement for semi-Lagrangian methods," Computers \& Fluids, vol. 46, pp. 180-185, 2011.

[2] Z. Liu and Y. He, "Solving the elliptic Monge-Ampère equation by Kansa's method," Engineering Analysis with Boundary Elements, vol. 37, no. 1, pp. 84-88, 2013.

[3] J. X. Hong, "The global smooth solutions of Cauchy problems for hyperbolic equation of Monge-Ampère type," Nonlinear Analysis: Theory, Methods \& Applications, vol. 24, no. 12, pp. 1649-1663, 1995.

[4] E. J. Dean and R. Glowinski, "Numerical methods for fully nonlinear elliptic equations of the Monge-Ampère type," Computer Methods in Applied Mechanics and Engineering, vol. 195, no. 1316, pp. 1344-1386, 2006.

[5] C. E. Gutiérrez, The Monge-Ampère Equation, Birkhäuser, Basel, Switzerland, 2011.

[6] O. C. Schnürer and K. Smoczyk, "Neumann and second boundary value problems for Hessian and Gauss curvature flows," Annales de l'Institut Henri Poincaré. Analyse Non Linéaire, vol. 20, no. 6, pp. 1043-1073, 2003.

[7] P.-L. Lions, N. S. Trudinger, and J. I. E. Urbas, "The Neumann problem for equations of Monge-Ampère type," Communications on Pure and Applied Mathematics, vol. 39, no. 4, pp. 539$563,1986$.

[8] J. Urbas, "The second boundary value problem for a class of Hessian equations," Communications in Partial Differential Equations, vol. 26, no. 5-6, pp. 859-882, 2001.

[9] J. Urbas, "Oblique boundary value problems for equations of Monge-Ampère type," Calculus of Variations and Partial Differential Equations, vol. 7, no. 1, pp. 19-39, 1998.

[10] J. Urbas, "On the second boundary value problem for equations of Monge-Ampère type," Journal für die Reine und Angewandte Mathematik, vol. 487, pp. 115-124, 1997.

[11] W. S. Zhou and S. Z. Lian, "The third initial-boundary value problem for an equation of parabolic Monge-Ampère type," Journal of Jilin University, no. 1, pp. 23-30, 2001.

[12] J. C. Kuang, Applied Inequalities, Shandong Science and Technology Press, 3rd edition, 2004.

[13] L. C. Evans, Partial Differential Equations, vol. 19 of Graduate Studies in Mathematics, American Mathematical Society, Providence, RI, USA, 1998.

[14] Y. Z. Chen, "Some methods of Krylov for the a priori estimation of solutions of fully nonlinear equations," Advances in Mathematics, vol. 15, no. 1, pp. 63-101, 1986.

[15] G. C. Dong, "Initial and nonlinear oblique boundary value problems for fully nonlinear parabolic equations," Journal of Partial Differential Equations. Series A, vol. 1, no. 2, pp. 12-42, 1988.

[16] O. A. Ladyženskaja, V. A. Solonnikov, and N. N. Ural'zeva, Linear and Quasilinear Equations of Parabolic Type, American Mathematical Society, 1995.

[17] C. Gerhardt, "Hypersurfaces of prescribed curvature in Lorentzian manifolds," Indiana University Mathematics Journal, vol. 49, no. 3, pp. 1125-1153, 2000. 